\title{
PRÁTICAS DE GESTÃo DE PESSOAS NAS EMPRESAS DO NORTE DO RIO GRANDE DO SUL
}

Eliana Andréa SEVERO ${ }^{1}$

Gisele Costa RABELLO ${ }^{2}$

Júlio César Ferro de GUIMARÃES ${ }^{3}$

\begin{abstract}
${ }^{1}$ Doutora em Administração pela Pontifícia Universidade Católica do Rio Grande do Sul, PUCRS, e pela Universidade de Caxias do Sul, UCS. Mestrado em Administração pela UCS. Especialização em Gestão Ambiental com Ênfase na Indústria pela UCS. Graduação em Administração pela Universidade Vale do Rio dos Sinos, UNISINOS. Graduação em Ciências Biológicas Licenciatura Plena pela Universidade de Santa Cruz do Sul, UNISC. Professora do Mestrado Acadêmico em Administração e da Graduação em Administração da Faculdade Meridional (IMED/RS). elianasevero2@hotmail.com

${ }^{2}$ Mestre em Administração pelo PPGA da IMED. MBA em Gestão de Pessoas. Graduação em Nutrição pela Universidade de Passo Fundo, UPF. gisele.rabello@hotmail.com

${ }^{3}$ Doutor em Administração pela Pontifícia Universidade Católica do Rio Grande do Sul, PUCRS, e pela Universidade de Caxias do Sul, UCS. Mestrado em Engenharia pela Universidade Federal do Rio Grande do Sul (UFRGS). Graduação em Administração pela UCS. Professor do Centro de Engenharias da UFPEL. juliocfguimaraes@yahoo.com.br
\end{abstract}

Recebido em: 26/10/2016 - Aprovado em: 10/09/2017 - Disponibilizado em: 30/12/2017

\section{RESUMO}

A gestão de pessoas trata-se de uma área estratégica, na qual se vincula os objetivos organizacionais e ações em prol das pessoas, sumarizados na visão, na missão e metas organizacionais.Perante o exposto, este estudo tem como objetivo analisar as práticas de gestão de pessoas em 29 organizações do norte do Rio Grande do Sul (RS).A metodologia utilizada tratou-se de uma pesquisa qualitativa, exploratória, por meio de um estudo de casos múltiplos. Os resultados destacam que a gestão de pessoas, enquanto prática estratégica, ainda é latente nas organizações analisadas, já que apenas a prática de recrutamento e seleção foi encontrada em todas as organizações. Contudo, espera-se uma atuação mais eficiente das empresas, promovendo programas de incentivo a qualidade de vida no trabalho, bem como o treinamento não apenas por necessidades emergenciais, que sejam realizados trabalhos contínuos de reciclagem e aprendizagem, pois influenciam positivamente a motivação e performance das organizações.

Palavras-chave: Gestão de pessoas. Práticas de gestão de pessoas. Importância. Benefícios. Organizações.

\section{PRACTICES OF HUMAN RESOURCE MANAGEMENT IN COMPANIES THE NORTHERN OF RIO GRANDE DO SUL}

\begin{abstract}
The management of human resources it is a strategic area, which is linked to organizational goals and actions for the benefit of people, summarized in the vision, mission and organizational goals. Given the above, this study aims to analyze the human resource management practices in 29 northern organizations of Rio Grande do Sul (RS). The methodology treated is a qualitative, exploratory, through a multiple case study. The results highlight tha the management of human resources as a strategic practice is still latent in the analyzed organizations, since only the practice of recruitment and selection found in all organizations. However, we expect a more efficient performance of enterprises by promoting programs to encourage the quality of working life and the training no tonly for emergency needs that continuous work of recycling and learning are carried out as positively influence the motivation and performance organizations.
\end{abstract}

Keywords: Human resource management. Human resource practices. Importance. Benefits. Organizations. 


\section{INTRODUÇÃO}

A gestão de pessoas trata-se de uma área estratégica, na qual se vincula os objetivos organizacionais e ações em prol das pessoas, sumarizados na visão, na missão e metas organizacionais.Neste contexto, a área de gestão de pessoas deve alinhara estratégia organizacional com o comprometimento organizacional, bem como as ações de qualidade de vida, as quais podem ocasionar um diferencial competitivo para as empresas (XHAKOLLARI, 2013; VENTURA; LEITE, 2014).

A região norte do Rio Grande do Sul (RS) possui um total de 357.823 habitantes e um total de Free On Board (FOB) U\$915.395.979 de exportações. Dentro desse cenário, está inserida a cidade de Passo Fundo, com um total de 196.587 habitantes, a qual possui um Produto Interno Bruto (PIB)per capita de $\mathrm{R} \$ 36.928,93$, sendo responsável por um total de exportação de U\$ FOB 878.124.847 (FEE, 2016), elencando extrema importância das organizaçõesinseridas nesta região.

Para que as empresas se mantenham competitivas, é necessário construir relações envolvendo as pessoas da organização e, consequentemente, promovendo resultados. Dessa forma, as práticas de gestão de pessoas (GP)têm papel fundamental no que tange o desenvolvimento dos funcionários no processo de mudança da organização.
Neste cenário, aGPtem como objetivo principal acompanhar as mudanças nesse ambiente competitivo e dinâmico para que se obtenha desempenho percebido pelo mercado através das pessoas (WRIGHT; GARDNER; MOYNIHAN, 2003). Nos últimos anos, esse papel tem sido amplamente discutido $\mathrm{e}$ também gerado algumas dúvidas quanto ao papel estratégico que o mesmo tem na organização. Neste contexto, as organizações possuem um departamento que controle os processos e políticas de recursos humanos, porém, muitas vezes não alinhados à estratégia da organização, e por vezes, sem práticas de GP que sustentem bons resultados.

Considerando que as empresas buscam adaptar-se às mudanças, destaca-se que a de GP deve ir além de executar seus processos básicos, realizando-os de forma efetiva e que esteja ligado à estratégia da organização, considerando o contexto externo ao qual está inserido e seus stakeholders (CORSO et al., 2014).Embora se tenha conhecimento da importância da GPe o desempenho organizacional e as relações positivas que existem entre as práticas de GP e seus resultados estratégicos, aindacarece-sede pesquisas que relacionam essas questões estratégicas às questões de GP (JABBOUR et al., 2012).

Perante o exposto, emerge a questão de pesquisa: quais as práticas de GP utilizadas pelas organizações do norte do RS? Neste sentido, este estudo tem como objetivo 
analisar as práticas de gestão de pessoas em 29 organizações do norte do RS. Além desta introdução, o artigo apresenta o referencial teórico elencando: a importância da gestão de pessoas para as organizações; as práticas de gestão de pessoas; metodologia utilizada; resultados e discussões; e, considerações finais.

\section{IMPORTÂNCIA DA GESTÃO DE PESSOAS PARA AS ORGANIZAÇÕES}

Embora a gestão de recursos humanos (GRH) exista há alguns anos e seja conceituada de maneiras diferentes em diversos países, somente quando deu-se início às ciências comportamentais é que a GRH teve seu valor evidenciado, criando assim, um foco estratégico para as práticas de GP(BREWSTER; MORLEY; BUCIUNIENE, 2010). Devido a isso, houve aumento da literatura sobre a temática de gestão de pessoas (GP)(HUANG, 2000), mas ainda há muito o que ser feito quando se trata em gerenciamento de pessoas (SCROGGINS; BENSON, 2010).

As pesquisas em GP foram publicadas durante os anos de 1980 e 1990, período esse que foi principalmente levantado os conceitos teóricos da temática. Após esse período, houve um avanço tanto no âmbito teórico, quanto no âmbito empírico da área(LENGNICK-HALL et al., 2009). Portanto, o período dos anos 2000 foi marcado por uma ampla compreensão da área, pois foram observados de maneira empírica as relações das práticas de GP com o desempenho organizacional (LENGNICKHALL et al., 2009;SCROGGINS; BENSON, 2010).

Destaca-se que os pesquisadores da área de GP estão mais atentos nas questões de implementação de práticas de GP para todos os envolvidos, a fim de garantir uma aplicação eficaz, através de indicadores que possibilitem bons resultados providos pelas pessoas à organização (LENGNICK-HALL et al., 2009).

A partir dessa evolução, a GPtem como papel principal a gestão efetiva das pessoas no contexto laboral a fim de garantir o sucesso de suas funções e responsabilidades, tornando o indivíduo cada vez mais satisfeito e produtivo (IVANCEVICH, 2009) e, ainda, conseguir influenciar positivamente no desempenho das organizações (JABBOUR et al., 2012).Além da organização ter um setor específico que crie e elabore as políticas e processos de gestão de GP, Ivancevich (2009) ressalta que o papel de gerir as pessoas de forma efetiva, também é papel do gestor direto, o qual deverá incentivar seus funcionários para o atingimento das metas tanto as gerais da organização, como também as metas individuais.

Conforme Ivancevich (2009) e Tachizawa (2015) a GP abrange diversas atividades relacionadas à vida das pessoas no 
contexto do trabalho e podem ser divididas oito atividades principais e mais comuns nas mais diversas organizações: (i) cumprimento das legislações referentes ao trabalho, (ii) análise de cargos, (iii) planejamento de recursos humanos, (iv) recrutamento, seleção, motivação e orientação de funcionários, (v) avaliação de desempenho e compensação, (vi) treinamento e desenvolvimento, (vii) relações trabalhistas, (viii) segurança, saúde e bemestar .

A GP dentro das organizações também está dividida em quatro dimensões, sendo elas: (i) direcionadas às ações, (ii) direcionada às pessoas, (iii) direcionada ao global e (iv) direcionada ao futuro (IVANCEVICH, 2009; WALL; WOOD, 2005). No que se refere a gestão de pessoas direcionada às ações, temse preocupação nas normas e procedimento escritos, concentrando-se nas ações relativas ao emprego. Já a gestão direcionada às pessoas, vê cada funcionário como um ser único, o qual merece uma ampla cobertura de serviços com foco em suprir a demanda de suas necessidades individuais. A gestão direcionada ao global define cada atividade com uma orientação globalizada, tratando todos com respeito e justiça, independente do local onde o funcionário está trabalhando, sendo que as normas e regras de $\mathrm{RH}$ são ajustadas conforme a realidade local. E, por fim, a gestão de RH direcionada ao futuro preocupa-se nas metas da organização, mas considera as pessoas como essenciais para atingir essas metas. Ainda nessa dimensão, a gestão deve incorporar em sua estratégia organizacional as pessoas como parte do processo (IVANCEVICH, 2009; WALL; WOOD, 2005).

Estudos com empresas americanas e alemãs demonstram que empresas que implementam práticas de GP que envolvam alto desempenho geram lucros significativos (IVANCEVICH, 2009) e geram valor às empresas (JIANG et al., 2012)).

A gestão estratégica de GP auxilia a organização gerando resultados positivos por meio de ações que: i) auxilia a organização a cumprir as metas estipuladas; ii) utilizar as competências necessárias para a realização das atividades adequadas; iii) funcionários devidamente treinados e motivados a desempenhar as atividades propostas ao cargo; iv) aumento da satisfação do empregado, através de ações de responsabilidade social, especialmente mantendo a qualidade de vida de todos os envolvidos(IVANCEVICH, 2009).

Em suma, o desafio encontrado pelas empresas vem sendo identificado no alinhamento de objetivos estratégicos com as principais práticas de GP(JABBOUR et al., 2012)e, para isso, no tópicos a seguir serão abordados as práticas de GP que contribuem para o atingimento de metas à estratégia organizacional. 


\section{PRÁTICAS DE GESTÃo DE PESSOAS}

Pesquisas têm demonstrado uma ampla compreensão de como as práticas de GP se relacionam com o desempenho da organização (GONG et al., 2009) e como e por quesão essenciais para trazer melhores resultados para as organizações (LACOMBE; ALBUQUERQUE, 2008). Dessa forma, continua atraindo a atenção de estudiosos, pois uma série de estudos ofereceram subsídios para examinar a relação entre as práticas de GP (LENGNICK-HALL et al., 2009).

$\mathrm{Na}$ pesquisa realizada por Khatri (2000) a qual examinou a relação entre estratégia, práticas de GP e desempenho da organização; os resultados mostraram que a estratégia global afeta as práticas de GP, bem como, as práticas de GP têm um efeito direto sobre o desempenho organizacional e, ainda, modera a estratégia de negócio de relacionamento entre as práticas de GP e o desempenho organizacional.

Na pesquisa de Huang et al. (2000) o desempenho organizacional é significativamente relacionado as práticas de GP, como: planejamento, recursos humanos, avaliação, remuneração, treinamento e desenvolvimento, indicando assim que empresas competitivas são aquelas que adotam uma abordagem altamente eficaz para a gestão dos recursos humanos presentes na organização.

Neste contexto, a pesquisa de Vlachos
(2008) destaca que as práticas de GP (segurança do emprego, recrutamento/seleção, equipes autogeridas e descentralização da tomada de decisões, remuneração; capacitação/treinamento e compartilhamento de informações) melhoraram o desempenho organizacional,

Gong et al. (2009) ressaltam que as práticas de GP destinadas a melhorar os conhecimentos, habilidades e capacidades dos funcionários contribuem para a melhoria de resultados da organização, gerando ainda, maior compromisso de todos. Dessa forma, os gestores podem aumentar de maneira significativa os benefícios em de investir nas práticas de $\mathrm{RH}$, motivando e desenvolvendo seus funcionários (JIANG 2012).

Estudos sugerem às organizações se concentraremnas práticas de GP (WHITE; BRYSON, 2013),pois representam o que realmente ocorre e se operacionaliza nas organizações no gerenciamento das pessoas (JABBOUR et al., 2012; JIANG et al., 2012), tais como recrutamento/seleção e treinamento para melhorar as habilidades dos funcionários(JIANG et al., 2012). Por outro lado, quando o objetivo é motivar os funcionários, é necessário remunerar baseado em desempenho, como forma de compensação pelas tarefas realizadas e retorno do trabalho(COLLINS; CLARK, 2003).

Há uma certa padronização nas práticas utilizadas pelas organizações na 
inclusão das práticas associadas à área de GP, como recrutamento, seleção, remuneração, avaliação de desempenho e demissão. Ainda, com menor frequência, observa-se a utilização de outras práticas de gestão, como padrão da comunicação, grau de participação e autonomia e desenho do trabalho (LACOMBE; ALBUQUERQUE, 2008).

Assim, as empresas tem se preocupado em avaliar e mensurar os resultados das práticas de GP, buscando melhorar as relações entre as estratégias organizacionais e as práticas, monitorando os custos e melhorando a sistemática de integração desses sistemas (JIANG et al., 2012; LACOMBE; ALBUQUERQUE, 2008; VLACHOS, 2008).

\section{METODOLOGIA UTILIZADA}

A metodologia utilizada neste estudo, tratou-se de uma pesquisa qualitativa, exploratória, por meio de um estudo de casos múltiplos, a fim de analisar as práticas de GP em 29 organizações do norte do RS. Conforme Malhotra et al. (2005) e Flick (2004), a pesquisa qualitativa proporciona uma melhor compreensão do contexto e do problema de pesquisa, auxiliando no refinamento de conceitos, na exploração de novas áreas de oportunidades e pesquisas sociais (GIBBS, 2009).

Segundo Creswell (2007) e Gil (2007), a pesquisa qualitativa pode ser caracterizada como exploratória, pois tem a finalidade de explorar um tópico, quando suas variáveis e bases teóricas são desconhecidas, objetivando a compreensão e a exploração de um conceito ou fenômeno.

Coerentemente, o escopo desta pesquisa trata-se como um estudo de caso múltiplo, uma vez que se trata de uma investigação empírica, pois os limites entre o fenômeno e o contexto não estão claramente definidos (YIN, 2005), realizado em 29 organizações, de diferentes portes, no norte do RS.

Para a coleta de dados utilizou-se a entrevista individual semiestruturadas. As entrevistas semiestruturadas têm como propósito descobrir as questões implícitas, onde o respondente é induzido a comentar sobre suas crenças, atitudes e informações subjacentes ao tema em estudo (MALHOTRA et al., 2005). O método de entrevistas individuais tem a oportunidade de explorar em profundidade um determinado problema de pesquisa, sendo o mais adequado para os objetivos proposto nesta etapa da pesquisa.

Os entrevistados foram os gestores responsáveis pela área de GP nas organizações.O roteiro da entrevista foi adaptado das pesquisas de Khatri (2000),Huang(2000) e Vlachos (2008), bem como validado por três experts da área temática de estudos.

As entrevistas ocorreram nos meses de abril 2015, maio e outubro de 2016. Posteriormente, para facilitar o processo de análise e interpretação, as entrevistas foram 
transcritas, conferidas com versão em áudio como forma de proporcionar precisão, fidelidade e interpretação dos dados (GIBBS, 2009). Por questões estratégias, o nome das empresas será preservado, sendo neste estudo representado por Empresa 1 (E1), Empresa 2 (E2) ... Empresa 29 (E29), bem como o nome dos entrevistados, sendo respectivamente identificados por Entrevistado 1 (ENT1), Entrevistado 2 (ENT2) ... Entrevistado 29 (ENT 29).

Para a análise e interpretação dos dados, utilizou-se a análise de conteúdo, de acordo com Bardin (2006), valendo-se as categorias a priori,relacionadas a GP, embasadas nos objetivos e no referencial teórico, sendo elas: i) elaboração de cargo; ii) recrutamento e seleção; iii) capacitação; iv) planos de remuneração; v) avaliação de desempenho; vi) saúde e segurança.

\section{RESULTADOS E DISCUSSÕES}

\section{Organizações analisadas}

As organizações analisadas estão apresentadas na Figura 1. Neste sentido, observa-se que foram encontrados diferentes portes de organizações: pequeno, médio e grande, relacionado ao faturamento anual da empresa: i) Micro Empresas (faturamento anual inferior a $\mathrm{R} \$ 360$ mil); ii) Empresas de Pequeno Porte (faturamento anual entre R\$360 mil e 3,6 milhões) (BRASIL, 2007); iii) Empresas de Médio Porte (faturamento anual entre $\mathrm{R} \$ 3,6$ milhões e $\mathrm{R} \$ 300$ milhões); iv) Empresas de Grande Porte (faturamento anual superior a $\mathrm{R} \$ 300$ milhões) (BRASIL, 2011). Neste contexto, 23empresas são do setor de comércio e serviços e 6 classificamse com indústrias.

Figura 1- Caracterização das empresas

\begin{tabular}{|c|c|c|c|c|}
\hline $\mathbf{E}$ & Setor & Porte & $\begin{array}{l}\text { Principais } \\
\text { atividades }\end{array}$ & $\begin{array}{l}\mathrm{N}^{\circ} \text { de } \\
\text { colab. }\end{array}$ \\
\hline E 1 & Com. & $\mathrm{M}$ & $\begin{array}{l}\text { Comercialização } \\
\text { de implementos } \\
\text { agrícolas e peças }\end{array}$ & 23 \\
\hline $\mathrm{E} 2$ & Ind. & $\mathrm{G}$ & $\begin{array}{l}\text { Indústria de } \\
\text { implementos } \\
\text { agrícolas }\end{array}$ & 563 \\
\hline E3 & Com. & $\mathrm{M}$ & $\begin{array}{l}\text { Venda por } \\
\text { atacado de } \\
\text { produtos } \\
\text { importados e } \\
\text { artigos de } \\
\text { vestuário } \\
\end{array}$ & 62 \\
\hline E4 & Com. & $\mathrm{P}$ & $\begin{array}{l}\text { Comercialização } \\
\text { de peças } \\
\text { agrícolas }\end{array}$ & 6 \\
\hline E 5 & Com. & M & $\begin{array}{l}\text { Distribuidora de } \\
\text { alimentos }\end{array}$ & 60 \\
\hline E6 & Serv. & $\mathrm{G}$ & Serviços de saúde & 3230 \\
\hline E7 & Serv. & $\mathrm{G}$ & Serviços de saúde & 1534 \\
\hline E 8 & Serv. & $\mathrm{M}$ & $\begin{array}{l}\text { Serviços de } \\
\text { crédito pessoal }\end{array}$ & 58 \\
\hline E9 & Serv. & $\mathrm{M}$ & $\begin{array}{l}\text { Distribuidora de } \\
\text { produtos }\end{array}$ & 26 \\
\hline E10 & Serv. & $\mathrm{M}$ & $\begin{array}{l}\text { Serviços e auto } \\
\text { peças }\end{array}$ & 81 \\
\hline E 11 & Com. & $\mathrm{M}$ & $\begin{array}{l}\text { Comércio de } \\
\text { cimento e } \\
\text { concreto }\end{array}$ & 31 \\
\hline E 12 & Com. & $\mathrm{P}$ & $\begin{array}{l}\text { Comércio de } \\
\text { produtos } \\
\text { alimentícios }\end{array}$ & 9 \\
\hline E 13 & Com. & $\mathrm{G}$ & $\begin{array}{l}\text { Comércio de } \\
\text { artigos para } \\
\text { vestuário }\end{array}$ & 34 \\
\hline E14 & Com. & $\mathrm{P}$ & $\begin{array}{l}\text { Comércio de } \\
\text { artigos para } \\
\text { vestuário }\end{array}$ & 5 \\
\hline E15 & Serv. & $\mathrm{G}$ & $\begin{array}{l}\text { Serviços } \\
\text { educacionais }\end{array}$ & 123 \\
\hline E 16 & Com. & $\mathrm{P}$ & $\begin{array}{l}\text { Comércio de } \\
\text { artigos para } \\
\text { vestuário }\end{array}$ & 11 \\
\hline E17 & Ind. & $\mathrm{P}$ & $\begin{array}{l}\text { Indústria de } \\
\text { medicamentos } \\
\text { veterinários }\end{array}$ & 15 \\
\hline E 18 & Ind. & $\mathrm{G}$ & $\begin{array}{l}\text { Indústria de } \\
\text { bebidas }\end{array}$ & 115 \\
\hline
\end{tabular}




\begin{tabular}{|c|c|c|c|c|}
\hline E19 & Serv. & $\mathrm{P}$ & $\begin{array}{l}\text { Distribuição de } \\
\text { alimentos e } \\
\text { produtos de } \\
\text { higiene }\end{array}$ & 11 \\
\hline E20 & Serv. & $P$ & $\begin{array}{l}\text { Edição integrada } \\
\text { à impressão de } \\
\text { jornais diários e } \\
\text { atividade de rádio }\end{array}$ & 134 \\
\hline E 21 & Com. & $\mathrm{M}$ & $\begin{array}{l}\text { Comércio de } \\
\text { materiais de } \\
\text { construção }\end{array}$ & 28 \\
\hline E 22 & Ind. & $\mathrm{P}$ & $\begin{array}{l}\text { Indústria de } \\
\text { medicamentos } \\
\text { homeopáticos } \\
\text { para animais }\end{array}$ & 17 \\
\hline E23 & Ind. & $G$ & $\begin{array}{l}\text { Indústria de } \\
\text { alimentos } \\
\text { derivados da } \\
\text { proteína animal }\end{array}$ & 3500 \\
\hline E24 & Com. & $\mathrm{P}$ & $\begin{array}{l}\text { Comércio } \\
\text { varejista de tintas } \\
\text { e material de } \\
\text { pintura }\end{array}$ & 5 \\
\hline E25 & Com. & $\mathrm{M}$ & $\begin{array}{l}\text { Distribuição de } \\
\text { produtos } \\
\text { alimentícios }\end{array}$ & 180 \\
\hline E26 & Com. & $G$ & $\begin{array}{l}\text { Comércio } \\
\text { varejista de } \\
\text { produtos } \\
\text { alimentícios }\end{array}$ & 1470 \\
\hline E 27 & Ind. & $\mathrm{P}$ & $\begin{array}{l}\text { Sementes para o } \\
\text { agronegócio }\end{array}$ & 29 \\
\hline E28 & Ind. & $G$ & $\begin{array}{l}\text { Indústria de } \\
\text { implementos } \\
\text { agrícolas }\end{array}$ & 167 \\
\hline E29 & Com. & $\mathrm{P}$ & $\begin{array}{l}\text { Comércio de } \\
\text { artigos para o } \\
\text { vestuário }\end{array}$ & 31 \\
\hline
\end{tabular}

Fonte: Elaborado pelos autores (2016).

\section{Práticas de gestão de pessoas}

A GP tem como objetivos planejar, implementar, controlar e coordenar as atividades relacionadas a elaboração de cargos, ao recrutamento, à seleção, à capacitação e qualificação, à elaboração de planos de remuneração e avaliação de desempenho, bem como as ações relacionadas à preservação da saúde e da segurança no ambiente de trabalho da organização (IVANCEVICH, 2009).
Neste contexto, a Figura 2 apresenta processos de GP (HUANG, 2000; VLACHOS， 2008; TACHIZAWA， 2015) utilizadas pelas empresas do norte do RS. Coerentemente, observa-se que muitas empresas não possuem um processo de GP totalmente estruturado(Figura 2),pois apenas 5 organizações apresentam as 6 práticas/processos de GP pesquisadas (GP1 elaboração de cargos, GP2 - recrutamento, GP3 - capacitação, GP4 - plano de remuneração, GP5 - avaliação de desempenho e G6 - saúde e segurança).

Figura 2 - Práticas de GP utilizadas

\begin{tabular}{|c|c|c|c|c|c|c|}
\hline $\mathbf{E}$ & GP1 & GP2 & GP3 & GP4 & GP5 & GP6 \\
\hline 1 & $\mathrm{X}$ & $\mathrm{x}$ & $\mathrm{x}$ & $\mathrm{x}$ & $\mathrm{X}$ & \\
\hline 2 & $\mathrm{x}$ & $x$ & $\mathrm{X}$ & $\mathrm{x}$ & $x$ & $\mathrm{x}$ \\
\hline 3 & $x$ & $\mathrm{X}$ & $\mathrm{X}$ & $\mathrm{x}$ & $x$ & \\
\hline 4 & & $\mathrm{x}$ & & & & \\
\hline 5 & $\mathrm{X}$ & $\mathrm{X}$ & $\mathrm{x}$ & $\mathrm{X}$ & $\mathrm{x}$ & \\
\hline 6 & $\mathrm{X}$ & $\mathrm{x}$ & $\mathrm{X}$ & $\mathrm{X}$ & & $\mathrm{x}$ \\
\hline 7 & $\mathrm{x}$ & $\mathrm{X}$ & $\mathrm{X}$ & $\mathrm{x}$ & & $\mathrm{x}$ \\
\hline 8 & $\mathrm{x}$ & $\mathrm{x}$ & $\mathrm{X}$ & $\mathrm{x}$ & & \\
\hline 9 & $\mathrm{x}$ & $\mathrm{X}$ & $\mathrm{X}$ & $\mathrm{x}$ & & \\
\hline 10 & $\mathrm{x}$ & $\mathrm{X}$ & $\mathrm{x}$ & $\mathrm{x}$ & $\mathrm{X}$ & \\
\hline 11 & $\mathrm{x}$ & $\mathrm{x}$ & $\mathrm{x}$ & $\mathrm{x}$ & & \\
\hline 12 & & $\mathrm{X}$ & & & & \\
\hline 13 & $\mathrm{x}$ & $\mathrm{x}$ & $\mathrm{x}$ & $\mathrm{X}$ & & \\
\hline 14 & $\mathrm{X}$ & $\mathrm{X}$ & $\mathrm{X}$ & & & \\
\hline 15 & $\mathrm{X}$ & $\mathrm{X}$ & $\mathrm{X}$ & $\mathrm{X}$ & & $\mathrm{x}$ \\
\hline 16 & $\mathrm{x}$ & $\mathrm{X}$ & & & & \\
\hline 17 & $\mathrm{x}$ & $\mathrm{x}$ & $\mathrm{X}$ & $\mathrm{X}$ & $\mathrm{X}$ & $\mathrm{x}$ \\
\hline 18 & $\mathrm{x}$ & $x$ & $x$ & $\mathrm{x}$ & $x$ & $\mathrm{x}$ \\
\hline 19 & $\mathrm{x}$ & $\mathrm{x}$ & $\mathrm{x}$ & & & \\
\hline 20 & & $\mathrm{x}$ & & & & \\
\hline 21 & $\mathrm{X}$ & $\mathrm{x}$ & $\mathrm{X}$ & $\mathrm{X}$ & & \\
\hline 22 & $x$ & $\mathrm{X}$ & $\mathrm{X}$ & $\mathrm{X}$ & & \\
\hline 23 & $\mathrm{x}$ & $x$ & $\mathrm{x}$ & $\mathrm{x}$ & & $\mathrm{x}$ \\
\hline 24 & & $\mathrm{x}$ & $\mathrm{X}$ & $\mathrm{X}$ & & \\
\hline 25 & $\mathrm{x}$ & $\mathrm{X}$ & $\mathrm{X}$ & $\mathrm{X}$ & & \\
\hline 26 & $\mathrm{x}$ & $\mathrm{x}$ & $x$ & $\mathrm{x}$ & & \\
\hline 27 & $\mathrm{x}$ & $\mathrm{X}$ & $\mathrm{x}$ & $\mathrm{x}$ & $\mathrm{X}$ & $\mathrm{X}$ \\
\hline 28 & $\mathrm{x}$ & $x$ & $\mathrm{x}$ & $\mathrm{X}$ & $x$ & $x$ \\
\hline 29 & $\mathrm{x}$ & $\mathrm{x}$ & $\mathrm{x}$ & $\mathrm{x}$ & & \\
\hline
\end{tabular}

Fonte: Elaborado pelos autores (2016). 


\section{Elaboração de cargos}

O processo de elaboração dos cargosé o primeiro item para as empresas se situarem internamente com suas atividades, é um processo que vai destacar os requisitos, responsabilidades e esforços conferidos ao ocupante do cargo, o que contribui para o bom desenvolvimento do processo de recrutar e selecionar pessoas.

Nas empresas pesquisadas, a elaboração de cargos é realizadapor diferentes perfis, por gestores de $\mathrm{RH}$, diretores,gerentes, estagiárias de psicologia, psicólogas da empresa, psicólogas terceirizadas e equipe de consultorias. Os cargos são delineados, desenhados, definidos e estabelecidos para se buscar a eficiência, a eficácia, a efetividade e a relevância organizacional.

(...) os cargos da empresa foram descritos pelos gestores do setor, pelos colaboradores e pelo RH. São revistas as descrições, quando em algum momento ocorre mudança das atividades (...) (ENT1).

(...) identificamos a rotina de trabalho para assim, definirmos a descrição de cargo de cada função (...) a descrição desses cargos foi atualizada há pouco tempo, antes não havia tudo tão bem definido. Quem projetou esse novo cenário foi o próprio diretor da empresa. $\mathrm{O}$ mesmo percebeu que seus funcionários estavam fazendo um pouco de tudo. Sendo assim, estabeleceu os cargos e tudo funciona mais profissional dessa forma (...) (ENT3).

(...) o projeto de descrição de cargos foi um projeto elaborado pelas estagiarias de psicologia da empresa, a fim de identificar a função dos trabalhadores na organização (...) (ENT5).

(...) os cargos foram descritos por uma empresa de consultoria (...) (ENT8).

(...) utilizamos uma ferramenta chamada Mapa de Competências, nele está descrito todas as atividades que o cargo exerce e as competências necessárias para tal. O mapa é elaborado pela empresa, pelo gerente de RH e gerente ou supervisor do cargo (...) (ENT9).
(...) a cada semestre os gestores recebem as descrições dos cargos e analisam, se houver alterações, informam ao RH (...) (ENT10).

(...) a própria empresa está realizando este processo. Está em fase de desenvolvimento (...) (ENT11).

(...) a análise de cargos é feita através de atividades e competências para cada setor. Os cargos estão descritos e foram elaborados pela própria empresa. As análises de descrição de cargos são feitas anualmente (...) (ENT17).

(...) a análise de cargos é realizada pelo setor de $\mathrm{RH}$ e a psicóloga que auxilia também nos testes de recrutamento e seleção (...) (ENT28).

Vale destacar, que algumas Empresas $(4,12,20$ e 24) não possuem modelos de desenhos de cargos. Tanto a Empresa 4 como a 12 e 24 são caracterizadas como pequeno porte e familiares, o que pode ter influenciado na decisão, pois a gestão é realizada pelos proprietários e familiares, assim como as demais funções da empresa. Entretanto, a Empresa 14 também se caracteriza como sendo de pequeno porte e familiar, e mesmo possuindo apenas 5 funcionários, a gestora elabora o modelo de desenho de cargos, esse modelo é clássico/tradicional, a partir da divisão do trabalho e fragmentação das tarefas definem-se os cargos. Coerentemente, o E14 ressaltou que esta abordagem de desenho de cargo emergiu da própria gestora, pois era aluna do Curso de Administração, a qual havia conhecido na disciplina de Gestão de Recursos Humanos a importância de se ter os cargos desenhados, atrelados as atividades e tarefas.

Neste contexto, a Empresa 20, que realiza a impressão de jornais diários e 
atividade de rádio, encontra-se a 49 anos no mercado, possui um Departamento de Pessoal, formado por uma gestora, e com 134 funcionários no quadro de colaboradores, não tem elaborado um modelo de desenho de cargos. A E20 ressaltou quando se necessita de um funcionário, a identificação é realizada pelo coordenador do setor, conforme a demanda de trabalho.

\section{Recrutamento e seleção}

Diferentes processos de recrutamento são utilizados pelas empresas. Todas as empresas pesquisadas utilizam recrutamento externo, tais como: jornais da cidade, rádio, agências de emprego, consultorias especializadas,Instituições de Ensino (IES), CIEE, CDL, psicólogas terceirizadas, redes sociais eSkype. Entretanto, emerge também o recrutamento interno, por meio de murais, do banco de dados das empresas, e-mail aos colaboradores e indicações de funcionários.

A Empresa 2 e 3 utilizam estratégias diferenciada para o recrutamento de cargos operacionais e de gestão. Na empresa 2, para os cargos operacionais são utilizados carros de som e o jornal local, já para os cargos gerenciais uma empresa terceirizada de recrutamento. Para as vagas de estágio é realizado uma parceria com uma IES local. Na Empresa 3, para a produção e montagem, solicita-se a indicação dos funcionários, logo após disponibiliza-se em agência de trabalho. Para cargos estratégicos utiliza-se as agências de emprego, bem como as redes sociais (Facebook).

No que se refere as formas de recrutamento elenca-se a entrevista, os testes comportamentais, testes psicológicos, de conhecimento específico, raciocínio lógico eproficiência língua estrangeira.

(...) as vagas operacionais são abertas e divulgadas na região através de carro de som que circulam nos bairros e pelo jornal local, que foram os meios mais eficientes para alcançar os objetivos (...) em relação as vagas de estágio elas são abertas e disponibilizadas à IES que são divulgadas nos cursos e tem sido uma estratégia bem-sucedida (...) dependendo da vaga, pode ocorrer uma prova de conhecimento, prova de proficiência em inglês, por exemplo. Tudo de acordo com o perfil da vaga $(. .).($ ENT2).

(...) nosso primeiro passo é analisar nosso banco de dados. Havendo pessoas dentro do perfil da vaga, chamamos para que os candidatos venham realizar o preenchimento do cadastro, onde também é realizado um teste de conhecimentos gerais, raciocínio lógico, algumas questões básicas de matemática, questões descritivas de opiniões sobre determinadas questões do dia a dia. E também é solicitado uma redação sobre seu histórico de vida (...) (ENT3).

(...) a empresa possui uma psicóloga terceirizada que faz a parte de necessidade de pessoal e recrutamento (ENT5).

(...) através do preenchimento de cadastro e entrevista de recrutamento (...) (ENT6).

(...) o RH entrevista a pessoa via ferramenta de Skype juntamente com o coordenador da vaga. Depois se selecionado duas pessoas, vamos para etapa de seleção (...) (ENT8).

(...) é feita divulgação de vagas por e-mail e murais, e, eventualmente utilizamos anúncios em rádios/jornais. Temos um vasto banco de dados, de onde são retirados a maioria dos candidatos (...) (ENT10).

(...) o processo de recrutamento ocorre inicialmente internamente na empresa. Caso não existam colaboradores aptos para a nova função, busca-se o banco de talentos interno ou recorre-se a um banco de talentos externo, com agências de emprego, SINE (...) (ENT11).

(...) a vaga é anunciada nos sítios de universidades, CIEE, redes sociais como Facebook, e com empresas parceiras, no caso do recrutamento externo (...) (ENT17). 
O processo de seleção busca entre os candidatos recrutados aqueles mais adequados aos cargos existentes na empresa, visando manter ou aumentar a eficiência e o desempenho do pessoal, bem como a eficácia da organização. Uma boa seleção costuma considerar não só a vaga atual, mas o potencial do candidato (WRIGHT; GARDNER; MOYNIHAN, 2003). Neste contexto, a Empresa 2 utiliza diferentes formas de seleção, elencando os cargos operacionais e estratégicos.

(...) para as vagas operacionais o processo se dá com o candidato se apresentando, com ou sem curriculum, preenche uma ficha de cadastro, em todas as terças e quintas-feiras pela manhã, passa por entrevista com setor de recrutamento, sendo aprovado, passa para uma entrevista com o gestor direto da área que demandou a vaga. E em algumas vagas ocorre teste prático, conforme o perfil da vaga e complexidade da tarefa operacional (..) as vagas estratégicas são processadas através de uma análise de curriculum, chama-se o candidato para entrevista, análise de perfil com testes psicológicos, e os pré-selecionados são encaminhados para a entrevista com o gestor direto da vaga (...) (ENT2).

(...) é realizado o preenchimento de uma ficha com os dados de identificação do candidato em questão, uma entrevista e a aplicação de alguns testes psicológicos (...) (ENT5).

Neste contexto, a Empresa 23, que produz alimentos derivados da proteína animal, e se trata de uma grande organização na região norte do $\mathrm{RS}$, com 3500 colaboradores,ressalta que devido a tipologia de sua área de produção, não necessita de avaliação psicológica e testes de conhecimentos, pois os funcionários atuam na linha de produção. A entrevistada também destacou que ocorre uma alta rotatividade no setor de produção, devido as tarefas repetitivas e manuais, o que acaba causando falta de motivação nos colaboradores.

(...) para a seleção é feita uma entrevista semiestruturada que disponibiliza o entrevistador formular outras perguntas ao longo da entrevista, essa etapa é a mais frequente nos processos seletivos, para uma maior obtenção de considerações pessoas quanto profissionais (...) dinâmica de grupo (...) não se usa muito avaliação psicológica e nem provas de conhecimento pois as vagas desta empresa não são necessárias. Após é encaminhado para o responsável que tomara a decisão final (...) (ENT23).

\section{Capacitação/treinamento}

Os

processos

de capacitação/treinamento são utilizados para melhorar o desempenho dos funcionários e a performance da organização. Ocorre diferentes formas de realizar o treinamento, entre os principais destacam-se o interno, por meio dos próprios gestores de cada área (feedback pessoal), de grupos de leituras, reuniões, especialistas nos assuntos, plataformas on-line, tele aulas, teatros de vendas, clientes ocultos e palestras in company. Destaca-se também a capacitação externa, realizado por empresas de consultorias.

O processo de treinamento é de extrema importância para qualquer porte de empresa. A Empresa 17, caracterizada como de microempresa, oferece treinamento a cada 20 dias, o gerente monta pequenas reuniões, apresenta pontos chaves e esclarece dúvidas de vários temas, como abordagens de atendimento aos clientes eo número de vendas para atingir objetivos e metas. 
Por apresentar uma tipologia de serviço diferente das demais empresas, a Empresa 15, fornecedora de serviços educacionais, oferece treinamento interno mensal, por meio da psicóloga organizacional. Este treinamento tem a duração de uma hora, divide-se a equipe de funcionários administrativos e de apoio pedagógico em duas equipes, manhã e tarde, a sistemática é feita inicialmente com um momento espiritual, dinâmica de grupo e reflexão em forma de vídeos e slides. O enfoque do treinamento é sobre as competências: benevolência, organização, visão sistêmica, trabalho em equipe, comprometimento, comunicação, relacionamento interpessoal, liderança, empreendedorismo e inovação. Contudo, também ocorrem dois encontros no retorno do semestre, e quem realiza é o Administrador, tendo o enfoque de atendimento ao cliente.

(...) $10 \%$ dos treinamentos disponibilizados pela empresa são internos e $90 \%$ externos. O treinamento interno é realizado pelos próprios colaboradores, o externo é em grande maioria disponibilizado por terceiros. Os treinamentos geralmente são treinamentos técnicos. Em 2014 treinamos 6.000hs (...) (ENT1).

(...) quando não há instrutor interno, busca-se no mercado o instrutor com a parceria de uma consultoria externa (...) (ENT2).

(...) temos grupos de leituras, onde colaboradores leem 4 livros ao ano. Livros que são direcionados pela Empresa. Após a leitura de cada livro, é feito um seminário onde o colaborador apresenta aos demais do grupo um resumo sobre seu livro (...) (ENT3).

(...) o último treinamento executado teve como público alvo os motoristas da empresa. Neste treinamento houve a criação de uma cartilha com os dez mandamentos dos motoristas e também a possibilidade de um curso de primeiros socorros (...) (ENT5).
(...) cada setor da instituição, através do RH, estabelece uma meta de horas de treinamento por funcionário, a qual deverá contemplar todas as atividades vinculadas ao desenvolvimento dos colaboradores. Em 2015 foi de 18h (...) (ENT6).

(...) atualmente os treinamentos são mais internos, realizados por especialistas no assunto, podendo ser alguém interno ou alguma consultoria ou parceria. Cada setor avalia a necessidade, planeja e registra o treinamento junto ao RH (...) (ENT7).

(...) ocorre conforme a demanda, externo e interno, o treinamento e fornecido por profissionais de instituições que fornecem cursos e também por uma empresa que presta assessoria em treinamentos para os agentes de crédito (...) (ENT8).

(...) a empresa possui cinco centros de treinamento na Matriz (...) possui uma plataforma interativa (com treinamentos on-line) e tele aulas (...) conforme as necessidade identificada pelos gestores (ENT10).

(...) acontece também treinamento com os professores, mas este item não faz parte da elaboração da psicóloga e sim da equipe pedagógica (...) (ENT15).

(...) recentemente a empresa implantou a Norma ISO 9001, na oportunidade, todos os funcionários passaram por um treinamento junto ao SEBRAE (...) (ENT17).

(...) a área responsável de treinamento anuncia o mesmo, e os funcionários que tiverem interesse se inscrevem. Os treinamentos praticamente são realizados todas as semanas, tanto internos como externos (depende do tema) (...) (ENT23).

\section{Planos de remuneração}

Uma política de remuneração adequada, consistente em relação às possibilidades da empresa e os valores praticados no mercado, trata-se de um importante instrumento de melhoria do nível de satisfação dos colaboradores, entretanto, não tece isoladamente responder pelos sucessos ou fracassos das políticas de gestão de pessoas(TACHIZAWA, 2015). Nas empresas pesquisadas, o plano de remuneração é elaborado pelo setor de $\mathrm{RH}$, ou pelo gestor responsável, por meio de tabela salarial e pesquisas regional. No caso da 
Empresa 7, prestadora de serviços de saúde, é realizada uma parceria com o Sindicato de Saúde, o qual define a classificação dos salários para a categoria.

(...) a escala de salário é determinada pela descrição de salários que é realizada através de tabela salarial, de cada função e de cada nível, conforme o seu conhecimento e o seu valor no mercado de trabalho (...) (ENT1).

(...) a organização não possui uma tabela salarial formal e estratégica, participamos da pesquisa salarial regional, principalmente para os cargos estratégicos. E esta pesquisa é utilizada para fazer as comparações e análises caso a caso (...) (ENT2).

(...) temos duas tabelas salariais: uma para colaborador e chefes de seção e outra para cargos gerenciais (...) (ENT3).

(...) a pesquisa salarial é feita pela empresa, com outros estabelecimentos hospitalares parceiros e juntamente com o sindicato (...) (ENT7).

Perante o exposto, a pesquisa verificou se nestes planos de remuneração, ocorria o modelo de remuneração por competências. Somente a Empresa 18 e 28 apresentam o modelo por competências, pois a 18 é uma multinacional, e traz uma expertise de gestão, que estimula e reconhece a aquisição e a disseminação do conhecimento por parte dos colaboradores,assim como a 28 que tem uma gestão que se preocupa com o desempenho e a qualidade de vida das pessoas, servindo como elemento de atração e retenção de talentos (IVANCEVICH, 2009).

No plano de remuneração, buscou-se os benefícios ofertados. No que tange os principais benefícios, destacam-se o vale alimentação eo plano de saúde. A participação nos lucros e resultados (PLR) foi encontrado nas Empresas 2, 17, 18, 27 e 28, assim como o auxílio educacional apenas nas Empresas 27 e 28. Neste cenário, destaca-se que as empresas da região norte do RS não evoluíram no quesito de benefícios, pois os mesmos ainda não são diversificadosem muitas das organizações analisadas.

\section{Avaliação de desempenho}

Para a implantação dos programas de avaliação, as organizações deverão definir os padrões de desempenho que serão avaliados e divulgados para todos os colaboradores.Cada empresa deve escolher o método mais adequado às suas realidades e cultura, ou desenvolve seu próprio sistema de avaliação (TACHIZAWA, 2015). Porém, o processo de avaliação de desempenho não é frequentemente utilizado pelas empresas pesquisadas.

No Caso da Empresa 8, o entrevistado ressaltou que mesmo se caracterizando como uma empresa prestadora de serviços de crédito pessoal, a qual tem metas mensais a serem alcançadas, o funcionário não é avaliado por meio das metas alcançadas, emerge uma avaliação mais subjetiva, elencando aspectos comportamentais. O E8 ainda ressalta que isto ocorre devido a falta de uma gestão de pessoas mais profissional, pois a empresa é familiar e governada pelos proprietários.

Entretanto, as Empresas 10, 18 e 28 realizam a avaliação de desempenho. A 
Empresa 10, por se tratar de uma concessionária de veículos, utilizada para a avaliação de desempenho o modelo padrão da montadora (Volkswagen), baseado nas competências. Já a Empresa 18 que é uma multinacional, realiza anualmente a avaliação de desempenho para o quadro operacional e técnico, esemestralmente para osgestores, bem como utiliza a avaliação de competências. Neste contexto, a Empresa 28 realiza a avaliação de desempenho com base nas competências para cada cargo, onde realiza-se uma avaliação 270 graus (auto avaliação, gestor, colega e cliente).

(...) como não há plano de carreira na empresa, planeja-se também ao contratar uma consultoria é também realizar construir o Plano de Carreira da Empresa 2 (...) (ENT2).

(...) mesmo tendo altas metas mensais para alcançar não temos um sistema de avaliação de desempenho, este está baseado mais em aspectos comportamentais, pois a empresa é familiar (...) (ENT8).

(...) a empresa faz avaliação de desempenho de forma informal (...) (ENT17).

(...) a avaliação é anual (...) é feita avaliação com operação e técnicos. Duas vezes ao ano com a liderança. A avaliação é feita pelo gestor e serve também como base para remuneração (...) (ENT18).

(...) a avalição é primordial para a gestão da empresa, utilizamos a avaliação por competências, 270 graus, o que impacta nas promoções e benefícios (...) (ENT28).

\section{Saúde e segurança}

No que tange as questões de saúde e segurança, apenas 9Empresas $(2,6,7,15,17$, $18,23,27$ e 28) utilizam práticas que visam eliminar efeitos que impactam a saúde e qualidade de vida dos funcionários. A disponibilização de EPIs foram as práticas mais utilizadas pelas empresas.
(...) temos descrito os EPIs necessário apara os cargos

(...) (ENT2)

(...) a equipe da CIPA elabora um treinamento duas vezes ao ano sobre o uso de EPI's (...) (ENT15).

(...) a área de produção utiliza os EPIs necessário a sua tarefa ou periculosidade (...) (ENT23)

Coerentemente, as Empresas que prestam serviços de saúde (6 e 7) possuem departamentos específicos que analisam os riscos de saúde e segurança, valem-se da utilização de EPIs, bem como de indicadores de acidentes de trabalho.

Neste contexto, o quesito de saúde e segurança, assim como a qualidade de vida no trabalho não é uma prática usual nas empresas norte do RS, estes são mais valorizados devido à necessidade ocasionada pelo risco inerente a execução da tarefa.

\section{CONSIDERAÇÕES FINAIS}

A área de GP elabora as políticas e processos de gestão, o que influência positivamente as organizações. Este estuado analisou as práticas de gestão de pessoas em 29organizações do norte do RS, bem como a sua importância para as organizações. Neste contexto, apenas 5 organizações apresentam práticas e processos estruturados de GP, que abordam desde a elaboração de cargos, recrutamento e seleção, capacitação, planos de remuneração, avaliação de desempenho, até a saúde e segurança, o que está de acordo com as pesquisas de Khatri (2000), Lacombe e Albuquerque (2008), Vlachos (2008), Ventura e Leite (2014) e Corso et al. 
(2014), pois ocasionam melhor integração dos sistemas de GP e vantagem competitiva para a organização.

Vale ressaltar que essas 5 organizações se classificam como indústrias, e destas, 2 tratam-se de organizações de grande porte, multinacionais, as quais importam o modelo de GP, visto que são organizações renomadas, preocupadas com as questões de saúde e segurança.Para Ivancevich (2009) e Xhakollari (2013), gerir as pessoas de forma efetiva prima para o comprometimento organizacional. Entretanto, a gestão de pessoas, enquanto prática estratégica,ainda é latente nas organizações analisadas, já que apenas a prática de recrutamento e seleção foi encontrada em todas as organizações.

Contudo, espera-se uma atuação mais eficiente das empresas, promovendo programas de incentivo a qualidade de vida no trabalho, bem como capacitação/treinamento não apenas por necessidades emergenciais, que sejam realizados trabalhos contínuos de reciclagem e aprendizagem, principalmente para as organizações prestadoras de serviços de saúde, na qual o desgaste psicológico é grande, por lidar com doenças humanas e pessoas.

Ao longo da pesquisa apresentaram-se algumas limitações, destacando-se principalmente no que se refere à sua capacidade de generalização, visto que foi explorada a realidade de 29 organizações, através da percepção de dois entrevistados.Por conseguinte, sugere-se uma análise mais abrangente, avaliando as práticas de GP a um número maior de empresas. Em acréscimo, são pertinentes estudos qualitativos e quantitativos que enfoquem a comparação entre regiões, setores, cadeias produtivas ou até mesmo a diferentes percepções entre diversos atores inseridos neste contexto.

\section{REFERÊNCIAS}

BARDIN, L. Análise de conteúdo. Lisboa: Edições 70, 2006.

BRASIL. 2007. Lei 11.638, de 28 de dezembro de 2007. Disponível em: <http://www.planalto.gov.br/ccivil_03/_ato20 07-2010/2007/lei/111638.htm>. Acesso em: 18 de mar. 2016.

BRASIL. 2011. Lei Complementar 139, de 10 de novembro de 2011. Disponível em:

http://www.receita.fazenda.gov.br/Legislacao/ LeisComplementares/2011/leicp139Acesso em: 10 de abr. 2016.

BREWSTER, C.; MORLEY, M.; BUCIUNIENE, I. The reality of human resource management in Central and Eastern Europe: a special issue to mark the 20th anniversary of Cranet (the Cran field Network on comparative human resource management). Baltic Journal of

Management, v. 5, n. 2, p. 145-155, 2010.

COLLINS, C. J.; CLARK, K. D. Strategic human resource practices, top management the role team social networks, and firm performance: of human resource practices in creating organizational competitive advantage. The Academy of Management Journal, v. 46, n. 6, p. 740-751, 2003.

CORSO, J. M.; PETRASKI, S. J.; SILVA, W. V.; TAFFAREL, M. Gestão estratégica de 
recursos humanos: identificando o processo de alinhamento estratégico. Tourism \& Management Studies, v. 10, n. Special, p. 49-57, 2014.

FEE - FUNDAÇÃO DE ECONOMIA E ESTATÍSTICA. 2015. Dipsonível em: $<$ http://www.fee.rs.gov.br/perfilsocioeconomico/municipios/detalhe/?municip io=Passo+Fundo $>$ Acesso em: 09 de Jul. de 2016.

GONG, Y.; LAW, K. S.; CHANG, S.; XIN, K. R.Human resources management and firm performance: the differential role of managerial affective and continuance commitment. The Journal of Applied Psychology, v. 94, n. 1, p. 263-275, 2009.

HUANG, T. C. Are thehumanresourcepracticesofeffectivefirmsdis tinctlydifferentfromthoseofpoorlyperformingo nes? Evidence from Taiwanese enterprises.

The International Journal of Human Resource Management, v. 11, n. 2, p. 436451, 2000.

IVANCEVICH, J. M. Gestão de recursos humanos. 10. ed. São Paulo: AMGH Editora Ltda., 2009.

JABBOUR, C. J. C.; FREITAS, W. R. de S.; TEIXEIRA, A. A.; JABBOUR, A. B. L. de S.Gestão de recursos humanos e desempenho operacional: evidências empíricas. Gestão de Produção, v. 19, n. 2, p. 347-360, 2012.

JIANG, K.; LEPAK, D. P.; HU, J.; BAER, J. C. How does human resource management influence organizational out comes? A metaanalytic investigation of mediating mechanisms. Academy of Management Journal, v. 55, n. 6, p. 1264-1294, 2012.

KHATRI, N. Managing human resource for competitive advantage: a study of companies in Singapore. The International Journal of Human Resource Management, v. 11, n. 2, p. 336-365, 2000.
LACOMBE, B. M. B.; ALBUQUERQUE, L. G. DE. Avaliação e mensuração de resultados em gestão de pessoas: um estudo com as maiores empresas instaladas no Brasil.

Revista de Administração da USP RAUSP, v. 43, n. 1, p. 5-16, 2008.

LENGNICK-HALL, M. L.; LENGNICKHALL, C. A.; ANDRADE, L. S.; DRAKE, B. Strategic human resource management: the evolution of the field. Human Resource Management Review, v. 19, n. 2, p. 64-85, 2009.

LEPAK, D. P.; LIAO, H.; CHUNG, Y.; HARDEN, E. E. A conceptual review of human resource management systems in strategic human resource management research. Research in Personnel and Human Resources Management, v. 25, n. 06, p. 217-271, 2006.

SCROGGINS, W. A.; BENSON, P. G. International human resource management: diversity, issues and challenges. Personnel Review, v. 39, n. 4, p. 409-413, 2010.

TACHIZAWA, T. Gestão com pessoas: uma abordagem aplicada às estratégias de negócios. 5 ed. Rio de Janeiro: FGV, 2015.

VENTURA, de S. L. V.; LEITE, P. R. N., Percepção da influência da gestão estratégica de pessoas no comprometimento organizacional. Pretexto, v. 15, n. 3, p. 11-28, 2014.

VLACHOS, I. The effect of human resource practices on organizational performance: evidence from Greece. The International Journal of Human Resource Management, v. 19, n. 1, p. 74-97, 2008.

XHAKOLLARI, L. Quality of work life of mental health professional in Albania.

Mediterranean Journal of Social Sciences, v. 4, n.1, p. 529-534, 2013.

WALL, T. D.; WOOD, S. J. The romance of human resource management and business performance, and the case for big science. 
Human Relations, v. 58, n. 4, p. 429-462, 2005.

WHITE, M.; BRYSON, A. Positive employee attitudes: how much human resource management do you need? Human

Relations, v. 66, n. 3, p. 385-406, 2013.
WRIGHT, P. M.; GARDNER, T. M.; MOYNIHAN, L. M. The impact of HR practices on the performance of business units. Human Resource Management Journal, v. 13, n. 3, p. 21-36, 2003. YIN, R. K. Estudo de caso: planejamento e métodos. 3. ed. Porto Alegre: Bookman, 2005. 